\title{
A Ligadura Tubária é Fator de Risco para a Redução da Densidade Mineral Óssea em Mulheres na Pós-menopausa?
}

\author{
Is Tubal Ligation a Risk Factor for a Reduction of Bone Mineral \\ Density in Postmenopausal Women?
}

\begin{abstract}
Silval Fernando Cardoso Zabaglia, Lúcia Helena Simões Costa-Paiva
Aarão Mendes Pinto-Neto
\end{abstract}

\begin{abstract}
RESUMO
Objetivo: comparar a densidade mineral óssea de mulheres menopausadas com e sem o antecedente de laqueadura tubária e avaliar quais fatores nos dois grupos poderiam estar associados à densidade mineral óssea no fêmur e coluna lombar.

Métodos: foram incluidas 70 pacientes em cada grupo, no ano de 1998, do Centro de Atenção Integral à Saúde da Mulher da Universidade Estadual de Campinas. As mulheres responderam um questionário sobre características clinicas e reprodutivas e foram submetidas à densitometria óssea (Lunar DPX) no fêmur e coluna lombar. Os dados foram analisados por meio dos testes t de Student, exato de Fisher, $\chi^{2}$ de Pearson, t com correção de Bonferroni e regressão múltipla.

Resultados: a média etária das laqueadas foi de 53,2 anos e das não-laqueadas de 52,6 anos, com média da idade à menopausa de 48 anos, semelhante nos dois grupos. As médias de idade à cirurgia foi 33,7 anos, com tempo decorrido da cirurgia de 18 anos. A média da densidade mineral óssea para o fêmur e coluna lombar não apresentaram diferenças significativas entre os dois grupos. A distribuição percentual em categorias de T-score do fêmur e coluna lombar não mostrou diferenças significativas nos dois grupos. Na regressão múltipla, observou-se que a idade mostrou uma associação inversa e o indice de massa corporal uma associação direta com a densidade mineral óssea no fêmur. Para a coluna lombar, cor não-branca, paridade, idade à menopausa, escolaridade e indice de massa corporal mostraram uma associação direta e a idade à menarca uma associação inversa com a densidade mineral óssea.

Conclusão: a laqueadura tubária não ocasionou redução na massa óssea em mulheres na pós-menopausa.
\end{abstract}

PALAVRAS-CHAVE: Contracepção. Osteoporose. Menopausa. Laqueadura tubária.

\section{Introdução}

A osteoporose é doença crônico-degenerativa caracterizada pela diminuição da massa óssea e deterioração da microarquitetura do tecido ósseo, tendo como conseqüência elevado risco de fratu$\mathrm{ra}^{1}$. É considerada a doença osteo-metabólica mais

Departamento de Tocoginecologia da Faculdade de Ciências Médicas da Universidade Estadual de Campinas Correspondência: Aarão Mendes Pinto Neto

Rua Alexander Fleming, 101 - Cidade Universitária "Zeferino Vaz"

13.083-970 - Campinas - SP

Fones (19) 3788-9306/3788-9402 - Fax (19) 3289-5935

e-mail: aarão@obelix.unicamp.br comum, pois afeta uma a cada três mulheres com idade superior a 65 anos, e até há alguns anos era considerada não-tratável e uma conseqüência inevitável da idade ${ }^{2-4}$.

No Brasil, dadas as dificuldades que cercam a realização de estudos epidemiológicos em um país de dimensões continentais, com uma complexa integração de diferentes padrões culturais e étnicos, ainda não existem números sobre a prevalência da osteoporose ${ }^{5}$. O estudo intitulado Osteoporose - Brasil ano 2000, que foi desenvolvido no país com cerca de 300 médicos especialistas, estimou que pouco mais de um terço dos doentes com osteoporose são diagnosticados e que, 
destes, somente $20 \%$ estão sendo submetidos a algum tipo de tratamento. Estas cifras indicam que a atenção à osteoporose encontra-se longe de ser uma situação controlada, caracterizando-se como um problema de saúde pública ${ }^{6}$.

Os principais fatores de risco para o desenvolvimento da osteoporose são o sexo feminino, o envelhecimento, a história familiar, raça branca, idade à menopausa e baixa estatura e peso. Fatores ambientais como a nutrição, o tabagismo, o sedentarismo e a ingestão de grandes quantidades de cafeína e álcool também podem influenciar relativamente a massa óssea ${ }^{7-12}$.

Apesar de esses fatores estarem estreitamente relacionados à massa óssea, a deficiência estrogênica pós-menopausa por si só é a principal causa de osteoporose em mulheres. Outras causas de hipoestrogenismo, como as secundárias a patologias endócrino-ginecológicas como amenorréia e hiperprolactinemia, ou pós-intervenção cirúrgica pélvica, como ooforectomia bilateral, também podem levar à diminuição de massa óssea ${ }^{13}$.

Outras cirurgias ginecológicas, como a laqueadura tubária e a histerectomia, podem afetar a circulação sangüínea ovariana em decorrência da ligadura da artéria tubária, que é ramo da artéria uterina, ou da própria artéria uterina ${ }^{14-16}$. Este comprometimento circulatório pode afetar a função ovariana e até levar à ocorrência de menopausa mais precocemente, com todas as outras conseqüências deste déficit hormonal precoce, incluindo a osteoporose ${ }^{11}$.

Observando-se a freqüência de ligaduras tubárias na população mundial e o aumento da incidência da osteoporose e das fraturas osteoporóticas em pacientes menopausadas, Fox e Cummings ${ }^{17}$ estudaram o papel da laqueadura tubária como um possivel fator de risco para diminuição da massa óssea e aumento no risco de fraturas, e demonstraram que as pacientes que apresentavam antecedente de laqueadura tubária tiveram uma pequena e não-significativa diminuição da densidade mineral óssea (DMO), sem aumento no risco de desenvolver fraturas.

Isto nos estimulou a realização deste estudo para avaliarmos se as mulheres brasileiras com antecedente de laqueadura tubária teriam um risco aumentado para o desenvolvimento da osteoporose, quando comparadas às pacientes nãolaqueadas.

\section{Pacientes e Métodos}

Foi realizado estudo do tipo corte transversal com 140 pacientes atendidas no Ambulatório de Menopausa do Departamento de Tocoginecologia do Centro de Atenção Integral à Saúde da Mulher (CAISM) da Universidade Estadual de Campinas (UNICAMP) no período de janeiro a dezembro de 1998, divididas em dois grupos: 70 pacientes laqueadas e 70 pacientes não-laqueadas.

Foram incluídas pacientes em amenorréia por um periodo de tempo superior a um ano, idade entre 45 e 65 anos e com conhecimento se foram ou não laqueadas.

Excluímos as pacientes com uso prévio de anticoncepcional hormonal oral por mais de 24 meses ou injetável por mais de 6 meses, presença de doenças crônicas como diabete melito, hipertiroidismo, hiperparatiroidismo, sindrome de má absorção, osteomalacia e neoplasias, antecedente de quimioterapia e/ou radioterapia e antecedentes de outras cirurgias pélvicas que não a laqueadura tubária: histerectomia, ooforectomia uni ou bilateral, salpingectomia uni ou bilateral. Também foram excluídas as que tivessem feito uso de medicamentos por mais de três meses que pudessem interferir com a massa óssea, como: cálcio, calcitonina, vitamina $\mathrm{D}$, hormônio tiroidiano, antitiroidianos, anticonvulsivantes, corticosteróides e terapia de reposição hormonal e as que fumassem mais de 20 cigarros por dia.

A variável independente estudada foi a laqueadura tubária. A variável dependente foi a DMO, quantificada na coluna lombar (L2-L4) e no fêmur proximal (colo do fêmur, triângulo de Wards e trocânter), sendo expressos em valores absolutos $\mathrm{em} \mathrm{g} / \mathrm{cm}^{2}$ : gramas de conteúdo mineral ósseo por área $\left(\mathrm{cm}^{2}\right)$ de osso analisado. A variável foi também descrita em valores relativos, em T-score (adulto jovem): comparação entre a DMO da paciente com a do pico de massa óssea esperado para adultos jovens e saudáveis do mesmo sexo. Os valores do $T$-score de cada grupo correspondem ao número de desvios padrão (DP) acima ou abaixo da média do grupo de referência (adulto jovem). Para a classificação, foram utilizados os critérios da WHO (1994) ${ }^{1}$ : normal - valores da DMO dentro de 1 DP da média do grupo padrão de referência; osteopenia - valores da DMO entre - 1 e -2,5 DP e osteoporose - valores da DMO 2,5 DP abaixo da média do grupo padrão de referência ${ }^{1}$.

As variáveis de controle analisadas foram: idade, cor, escolaridade, tabagismo, paridade, idade à menarca, tempo de menopausa, idade da paciente no momento da cirurgia, tempo decorrido desde a cirurgia e índice de massa corporal (IMC).

Inicialmente foram elaboradas tabelas descritivas utilizando-se média e desvio padrão, sendo a comparação das médias realizadas através do teste $t$ de Student. Para a comparação das freqüên- 
cias, foram utilizadas os testes exato de Fisher e $\chi^{2}$ de Pearson. Definiu-se como significativo o valor de $\mathrm{p}<0,05$.

Para a comparação das médias da DMO em $\mathrm{g} / \mathrm{cm}^{2}$ no fêmur foram utilizados o teste $t$ com correção de Bonferroni, que faz uma comparação múltipla dos três locais analisados, e o teste $t$ de Student. Para coluna lombar, utilizou-se o teste $t$ de Student para amostras independentes. Para análise da distribuição segundo categorias do $T$ score, normal ou alterada, de acordo com os locais analisados, utilizou-se o teste $\chi^{2}$ de Pearson.

Para a análise de regressão linear múltipla foram estudadas as variáveis idade, cor, escolaridade, tabagismo, paridade, idade à menarca, idade à menopausa, tempo de menopausa e índice de massa corporal.

O projeto de pesquisa deste estudo foi avaliado e aprovado pela Comissão de Pesquisa do Departamento de Tocoginecologia e Comitê de Ética da Faculdade de Ciências Médicas da UNICAMP.

\section{Resultados}

As pacientes com e sem antecedente de laqueadura tubária foram semelhantes em relação à média etária, idade à menarca, idade à menopausa, tempo médio de menopausa e IMC. As pacientes com laqueadura tubária haviam sido submetidas à cirurgia há 18,8 anos em média (mínimo de 6 anos, máximo de 32 anos), com média de idade quando da cirurgia de 33,7 anos (mínimo de 22 anos, máximo de 47 anos) (Tabela 1).

Tabela 1 - Distribuição das características das pacientes segundo o antecedente de laqueadura tubária.

\begin{tabular}{lrrrrr}
\hline Variáveis (anos) & \multicolumn{5}{c}{ Laqueadura tubária } \\
& Sim $(\mathbf{n}=\mathbf{7 0})$ & \multicolumn{2}{c}{ Não $(\mathbf{n}=\mathbf{7 0})$} & $\mathbf{p}^{*}$ \\
& Média & DP & Média & DP & \\
\hline Idade & 53,2 & 4,1 & 52,6 & 3,8 & 0,65 \\
Menarca & 13,2 & 1,8 & 13,2 & 1,6 & 0,88 \\
Menopausa & 48,0 & 3,0 & 48,9 & 3,4 & 0,11 \\
Tempo de menopausa & 4,4 & 3,3 & 3,7 & 2,9 & 0,23 \\
IMC & 26,2 & 3,3 & 29,7 & 3,0 & 0,30 \\
Idade à cirurgia & 33,7 & 5,9 & - & & - \\
Tempo desde a cirurgia & 18,8 & 6,1 & - & & - \\
\hline
\end{tabular}

* teste $t$ de Student

IMC, índice de massa corporal

A maioria das pacientes era branca e nãofumante. Aproximadamente dois terços das pacientes relataram escolaridade de até quatro anos e eram, na maioria, multíparas. A distribuição das variáveis avaliadas foi semelhante nos dois grupos (Tabela 2).

Tabela 2 - Características pessoais e antecedentes obstétricos das pacientes segundo 0 antecedente de laqueadura tubária.

\begin{tabular}{|c|c|c|c|c|c|}
\hline \multirow[t]{3}{*}{ Variáveis } & \multicolumn{4}{|c|}{ Laqueadura tubária } & \multirow[b]{3}{*}{$p^{*}$} \\
\hline & \multicolumn{2}{|c|}{$\operatorname{Sim}(n=70)$} & \multicolumn{2}{|c|}{ Não (n = 70) } & \\
\hline & $\mathrm{n}$ & $\%$ & $\mathrm{n}$ & $\%$ & \\
\hline \multicolumn{6}{|l|}{ Cor } \\
\hline Branca & 68 & 97,1 & 66 & 94,3 & $0,68^{*}$ \\
\hline Não-branca & 2 & 2,9 & 4 & 5,7 & \\
\hline \multicolumn{6}{|l|}{ Tabagismo } \\
\hline Sim & 7 & 10,0 & 9 & 12,9 & $0,60^{* *}$ \\
\hline Não & 63 & 90,0 & 61 & 87,1 & \\
\hline \multicolumn{6}{|l|}{ Escolaridade } \\
\hline Sem escolaridade & 4 & 5,7 & 4 & 5,7 & $0,86^{* *}$ \\
\hline 1 a 4 anos & 44 & 62,9 & 48 & 68,6 & \\
\hline 5 a 8 anos & 14 & 20,0 & 10 & 14,3 & \\
\hline 9 anos ou mais & 8 & 11,4 & 8 & 11,4 & \\
\hline \multicolumn{6}{|l|}{ Paridade } \\
\hline Nulíparas & 1 & 1,4 & 4 & 5,7 & $0,25^{*}$ \\
\hline 1 a 3 partos & 38 & 54,3 & 42 & 60,0 & \\
\hline Mais que 3 partos & 31 & 44,3 & 24 & 34,3 & \\
\hline Total & 70 & 100,0 & 70 & 100,0 & \\
\hline
\end{tabular}

A comparação das médias da DMO em $\mathrm{g} / \mathrm{cm}^{2}$, avaliadas ao nivel do fêmur e coluna lombar das pacientes com e sem antecedente de laqueadura tubária, não mostrou diferenças estatisticamente significativas entre os dois grupos (Tabela 3).

Tabela 3 - Média da densidade mineral óssea $\left(\mathrm{g} / \mathrm{cm}^{2}\right)$ no fêmur e coluna lombar das pacientes segundo 0 antecedente de laqueadura tubária.

\begin{tabular}{|c|c|c|c|c|c|}
\hline \multirow[t]{3}{*}{ Local } & \multicolumn{4}{|c|}{ Laqueadura tubária } & \multirow{3}{*}{$p$} \\
\hline & \multicolumn{2}{|c|}{$\operatorname{Sim}(n=70)$} & \multicolumn{2}{|c|}{ Não (n = 70) } & \\
\hline & Média & DP & Média & DP & \\
\hline \multicolumn{6}{|l|}{ Fêmur } \\
\hline Colo & 0,94 & 0,14 & 0,92 & 0,13 & $1,00^{*}$ \\
\hline Wards & 0,81 & 0,16 & 0,79 & 0,13 & $0,87^{*}$ \\
\hline Trocânter & 0,78 & 0,13 & 0,76 & 0,12 & $0,51^{*}$ \\
\hline \multicolumn{6}{|l|}{ Coluna lombar } \\
\hline $\mathrm{L}_{2}-\mathrm{L}_{4}$ & 1,06 & 0,13 & 1,07 & 0,19 & $0,28^{* *}$ \\
\hline
\end{tabular}

A distribuição percentual em categorias de T-score mostrou que não houve diferenças signifi- 
cativas nas porcentagens de densitometrias normais e alteradas nos dois grupos, em nenhum dos locais do fêmur. Em relação à coluna lombar, cerca de $60 \%$ das pacientes não laqueadas e $44 \%$ das pacientes laqueadas apresentaram diminuição de densidade óssea; entretanto, essa diferença não foi estatisticamente significativa (Tabela 4).

Tabela 4 - Distribuição percentual das pacientes com e sem antecedente de laqueadura tubária segundo as categorias de $T$-score do fêmur e coluna lombar.

\begin{tabular}{|c|c|c|c|c|c|}
\hline \multirow[t]{3}{*}{ Local } & \multicolumn{5}{|c|}{ Laqueadura tubária } \\
\hline & \multicolumn{2}{|c|}{$\operatorname{Sim}(n=70)$} & \multicolumn{2}{|c|}{ Não $(n=70)$} & \multirow[t]{2}{*}{$\mathrm{p}^{*}$} \\
\hline & $\begin{array}{c}\text { Alterado } \\
(<-1,0)\end{array}$ & $\begin{array}{l}\text { Normal } \\
(>-1,0)\end{array}$ & $\begin{array}{c}\text { Alterado } \\
(<-1,0)\end{array}$ & $\begin{array}{l}\text { Normal } \\
(>-1,0)\end{array}$ & \\
\hline \multicolumn{6}{|l|}{ Fêmur } \\
\hline Colo & 31,4 & 68,9 & 30,0 & 70,0 & 0,86 \\
\hline Wards & 48,6 & 51,4 & 45,7 & 54,3 & 0,74 \\
\hline Trocânter & 22,9 & 77,1 & 27,1 & 72,9 & 0,56 \\
\hline \multicolumn{6}{|c|}{ Coluna lombar } \\
\hline $\mathrm{L}_{2}-\mathrm{L}_{4}$ & 44,3 & 55,7 & 60,0 & 40,0 & 0,06 \\
\hline
\end{tabular}

$\mathrm{Na}$ análise de regressão linear múltipla, o IMC mostrou uma associação direta com a DMO para os locais do fêmur avaliados. A idade mostrou uma associação inversa com a DMO do colo do fêmur. Para a coluna lombar, as variáveis diretamente associadas à DMO foram o IMC, a cor nãobranca, paridade, idade à menopausa e a escolaridade, ao passo que a idade à menarca mostrou uma associação inversa. A laqueadura tubária não se associou à DMO nos locais avaliados.

\section{Discussão}

A principal proposição deste estudo foi avaliar se pacientes que foram submetidas à laqueadura tubária apresentam densidade mineral óssea inferior àquelas que não foram submetidas a este procedimento cirúrgico. Representa uma tentativa de verificar se as mulheres laqueadas apresentavam maior risco de desenvolver osteoporose que aquelas não-laqueadas. Os resultados demonstraram que não houve diferenças estatísticas significativas entre a DMO na coluna lombar e no fêmur das pacientes submetidas à cirurgia comparadas à das pacientes não esterilizadas cirurgicamente.

Os resultados estão de acordo com os relatados por Fox e Cummings ${ }^{17}$, que estudaram mulheres com mais de 65 anos e não observaram dife- renças significativas na densidade óssea daquelas com o antecedente de laqueadura tubária em relação às que não apresentavam este antecedente.

Os resultados do presente estudo fazem supor que realmente a cirurgia não produziu alteração circulatória ou disfunção hormonal capaz de interferir na massa óssea de mulheres climatéricas. Esta constatação está de acordo com as observações de Hobeika et al. ${ }^{18}$, que também avaliaram os efeitos de uma cirurgia de maior porte, a histerectomia com conservação ovariana bilateral realizada durante a menacme, que poderia teoricamente afetar a função ovariana. Estes autores no entanto não observaram diferenças significativas na densidade óssea do fêmur e coluna lombar, em pacientes com e sem o antecedente de histerectomia. Entretanto, outros estudos descreveram uma diminuição da densidade óssea em mulheres submetidas à histerectomia com conservação ovariana ${ }^{15,19-21}$. Menon et al. ${ }^{21}$ observaram que mulheres histerectomizadas com conservação ovariana apresentaram quadro de hipoestrogenismo suficiente para causar ondas de calor, perda óssea e elevação das concentrações sangüineas de ácido úrico. Esses resultados contraditórios na literatura relacionados ao déficit estrogênico secundário a situações como cirurgias pélvicas corroboram os de Torgerson et al. ${ }^{22}$, que afirmaram que o rastreamento das populações, por meio da identificação de outros fatores associados ao hipoestrogenismo levando à osteoporose, continua sendo um tema que merece investigação.

Além da deficiência estrogênica e da idade, que estão intimamente relacionadas à densidade óssea, outros fatores têm sido muito estudados e estão bem caracterizados. O que não se sabe com exatidão é a contribuição isolada de cada um durante o crescimento e particularmente sobre suas interações, influenciando a massa óssea final ${ }^{23,24}$.

$\mathrm{Na}$ presente casuística, as pacientes foram muito semelhantes em relação às variáveis de controle. Especificamente em relação à idade na qual a ligadura tubária foi realizada (média de 33,7 anos), é muito provável que toda a massa óssea já estivesse formada e, mesmo tendo havido pequenas lesões na circulação ovariana, estas não foram suficientes para trazer conseqüências esqueléticas futuras.

O peso corporal é um dos determinantes mais importantes da massa óssea individualmente nas mulheres. Isto é uma verdade parcial, pois, quando se ajustam os dados do peso com a estatura, sua influência diminui em aproximadamente $10 \%^{24,25}$. Nesta série de mulheres, com pelo menos 53 anos, não observamos diferenças significativas em relação ao índice de massa corporal 
entre os grupos, o que minimiza um viés importante em relação a estas variáveis.

Neste estudo, não houve diferenças quanto à cor das pacientes avaliadas nos dois grupos. Também não se observaram diferenças na escolaridade, que pode ser considerada como indicativa de estrato sociocultural e, conseqüentemente, do padrão de ingestão de alimentos, especialmente os ricos em cálcio.

A análise de regressão múltipla confirmou que o antecedente de laqueadura tubária não se associou a alterações na massa óssea, tanto no fêmur como na coluna lombar. Estes resultados ressaltam a importância do tema, pois vivemos uma verdadeira epidemia de esterilizações cirúrgicas femininas, muitas vezes realizadas sem considerar os critérios de elegibilidade dos métodos anticoncepcionais, gerando descontentamento e, outras vezes, problemas de saúde para muitas mulheres.

As leis brasileiras, aprovadas pelo Congresso Nacional em 1997, dispõem que quanto ao critério de elegibilidade para laqueadura tubária somente as mulheres com mais de 25 anos e com pelo menos dois filhos poderiam ser candidatas à cirurgia para esterilização definitiva $^{26}$. É importante ressaltar que, pelo menos do ponto de vista da osteoporose, um possivel comprometimento da função ovariana, decorrente da cirurgia realizada nesta faixa etária, é certamente muito precoce.

A confirmação de um maior risco de desenvolvimento de osteoporose futura em mulheres laqueadas poderia contribuir para a diminuição no número dessas cirurgias em nosso meio. Sendo assim, outros estudos abordando este tema ainda são necessários, de preferência prospectivos, com medidas da DMO ao longo do tempo, na menacme, antes e após a laqueadura, e com controle rigoroso de variáveis confundidoras, para melhor esclarecimento de influência da laqueadura tubária sobre o risco de osteoporose futura em dois grupos de mulheres, com e sem o antecedente da cirurgia.

\section{SUMMARY}

Purpose: to compare bone mineral density (BMD) of postmenopausal women with and without the antecedent of tubal ligation, as well as to evaluate the associated factors that affect the bone mineral density of femur and lumbar spine of both groups.

Methods: 70 postmenopausal women in each group were analyzed at CAISM-UNICAMP, during the year of 1998.
All women answered a questionnaire about some clinical and reproductive characteristics and underwent bone densitometry (Lunar DPX) to measure bone mineral density of the femur and lumbar spine. Statistical analyses were performed using Student's t-test, Fisher's exact test, Pearson $\chi^{2}$ test, Bonferroni 's test and multiple regression analysis.

Results: mean age of patients was 53.2 years and for controls it was 52.6 years. Mean age of 48 years at menopause was similar in both groups. Mean age at tubal ligation surgery was 33.7 years and time since surgery was 18 years. The multiple comparison of the average bone mineral density of femur and lumbar spine did not show statistical differences between the groups. The percentage distribution of the Tscore categories of femur and lumbar spine, classified into normal and altered, also did not show statistical differences between both groups. Multiple regression analysis showed that bone mineral density of femur was directly associated with body mass index, but age was indirectly associated. The variables dark skin, parity, age at menopause, educational level and body mass index were directly associated with bone mineral density of lumbar spine, but age at menarche was inversely associated.

Conclusion: these findings suggest that tubal ligation does not seem to cause an additional reduction in bone mineral density when evaluated in postmenopause.

KEY WORDS: Contraception. Osteoporosis. Menopause. Tubal ligation.

\section{Referências}

1. WHO Study Group. Assessment of fracture risk and its application to screening for postmenopausal osteoporosis. Geneva: World Health Organization; 1994. p.1-129. (Technical Report Series, 843).

2. Riggs BL. Pathogenesis of osteoporosis. Am J Obstet Gynecol 1987; 156:1342-6.

3. Gil-Antuñano SP, Ceño CM. Epidemiología, clínica, diagnóstico y screening de la osteoporosis postmenopausica. In: Gil-Antuñano SP, editor. Climaterio y menopausia: fascículo $2.1^{\mathrm{a}}$ ed. Madrid: Mirpal; 1993. p.129-36.

4. Szejnfeld VL, Rodrigues de Lima G, Atra E. Osteoporose no climatério. In: Pinotti JA, Halbe HW, Hegg R, editores. Menopausa. $1^{\text {st }}$ ed. São Paulo: Rocca; 1995. p.189-211.

5. Säo Paulo (Estado). Secretaria da Saúde. Hospital do Servidor Público Estadual. Osteoporosis 1995: basic diagnosis and therapeutic elements for a National Consensus Proposal. São Paulo Med J 1995; 113 Suppl: 1-64.

6. Marques Neto JF, Lederman R. Osteoporose: Brasil ano 2000. $1^{\text {st }}$ ed. São Paulo: Limay; 1995. 
7. Cummings SR, Kelsey JL, Nevitt MC, O’Dowd KJ. Epidemiology of osteoporosis and osteoporotic fractures. Epidemiol Rev 1985; 7:178-208.

8. Geusens P, Dequeker J, Verstraeten A, Nijs J. Age, and sex-, and menopause-related changes of vertebral and peripheral bone: population study using dual and single photon absorptiometry and radiogrammetry. J Nucl Med 1986; 27:1540-9.

9. Rae MH, Mole PA, Paterson CR. Endogenous factors affecting bone mineral content in postmenopausal women. Maturitas 1991; 13:319-24.

10.Curiel MD. Fisiopatología de la osteoporosis postmenopáusica In: Gil-Antuñano SP, editor. Climaterio y menopausia: fascículo $2.1^{\mathrm{a}} \mathrm{ed}$. Madrid: Mirpal; 1993. p.119-28.

11.Fernandes CE, Wehba S, Melo NR. Osteoporose pós-menopáusica. Femina 1996; 24 Supl:3-26.

12.Eastell R. Treatment of postmenopausal osteoporosis. N Engl J Med 1998; 338:736-46.

13.Riggs BL, Melton LJ III. Osteoporosis: etiology, diagnosis, and management. $2^{\text {nd }}$ ed. Philadelphia: Lippincott - Raven; 1995.

14. Camargo SF. Cirurgia ginecológica: propostas e refinamentos. $2^{a}$ ed. São Paulo: Fundo Editorial BYK; 1998. A cirurgia dos ovários: operar? O quê, quando, como? Iatrogenia ou profilaxia? p.181-186.

15.Hreshchyshyn MM, Hopkins A, Zylstra S, Anbar M. Effects of natural menopause, hysterectomy and oophorectomy on lumbar spine and femoral neck bone densities. Obstet Gynecol 1988; 72:631-8.

16.Oldenhave A, Jaszmann LJ, Everaerd WT, Haspels AA. Hysterectomized women with ovarian conservation report more severe climacteric complaints than do normal climacteric women of similar age. Am J Obstet Gynecol 1993; 168:765-71.

17.Fox KM, Cummings SR. Is tubal ligation a risk factor for low bone density and increased risk of fracture? Am J Obstet Gynecol 1995; 172:101-5.
18. Hobeika JD, Pinto-Neto AM, Costa-Paiva LHS, Pedro AO, Martinez EZ. Densidade mineral óssea de mulheres na pós-menopausa com e sem antecedente de histerectomia com conservação ovariana bilateral. Rev Bras Ginecol Obstet 2000; 22:475-9.

19.Simões RD, Baracat EC, Szjenfeld VL, Lima GR, Gonçalves WJ, Bortoletto CCR. Effects of simple hysterectomy on bone loss. São Paulo Med J 1995; 113:1012-6.

20.Watson NR, Studd JW, Garnett T, Savvas M, Milligan P. Bone loss after hysterectomy with ovarian conservation. Obstet Gynecol 1995; 86:72-7.

21. Menon RK, Okonofua FE, Agnew JE, et al. Endocrine and metabolic effects of simple hysterectomy. Int J Gynaecol Obstet 1987; 25:459-63.

22.Torgerson DJ, Donaldson C, Russell IT, Reid DM. Hormone replacement therapy: compliance and cost after screening for osteoporosis. Eur J Obstet Gynecol Reprod Biol 1995; 59:57-60.

23.Kelly PJ, Eisman JA, Sambrook PN. Interaction of genetic and environmental influences on peak bone density. Osteoporos Int 1990; 1:56-60.

24.Heaney RP, Matkovic V. Inadequate peak bone mass. In: Riggs BL, Melton LJ III, editors. Osteoporosis: etiology, diagnosis, and management. $2^{\text {nd }}$ ed. Philadelphia: Lippincott - Raven; 1995; p115-131.

25. Reid IR, Plank LD, Evans MC. Fat mass in an important determinant of whole body bone density in premenopausal women but not in men. J Clin Endocrinol Metab 1992; 75:779-82.

26. Brasil. Lei $n^{\circ} 9.263$ de 12 de janeiro de 1996. Regulamenta o parágrafo 7 do artigo 226 da Constituição Federal que trata do planejamento familiar, estabelece penalidades e dá outras providências. Diário Oficial da União, Brasília, 15 jan 1996. Seção I, n. 159, p. 17.989.

\section{RBGO}

\section{é uma publicação da \\ FEBRASGO}

e que aceita artigos provenientes de ginecologistas, obstetras e de outras especialidades. Portanto, publique!!! 